\title{
Desarrollo urbano sostenible en la agenda de cohesión territorial europea: Política de Ciudades Polis XXI, Portugal ${ }^{1}$
}

\author{
Sustainable Urban Development in the European Territorial \\ Cohesion Agenda: Polis XXI Policy, Portugal
}

\section{Margarida Queirós ${ }^{2}$}

\section{Resumen}

En este artículo se reflexiona sobre las políticas de desarrollo urbano sostenible en Europa y sus reflejos en Portugal, dando especial enfoque a la Política de Ciudades Polis XXI portuguesa, como un instrumento que tiende a seguir recientes orientaciones de política territorial y urbana en Europa. Se procura demostrar que este es un instrumento innovador y multidimensional de desarrollo urbano sostenible, estimulador de procesos interactivos de coordinación y agregación de actores en torno de objetivos y proyectos comunes (arreglos de gobernanza), señalando que las políticas de ordenación territorial están tendiendo hacia la institucionalización, cooperación y cohesión territorial. A partir de una selección de experiencias resultantes de la aplicación del instrumento Política de Ciudades Polis $X X I$, en Portugal, se busca concluir sobre cuáles son sus efectos y qué aspectos son cruciales para transferencias de conocimiento y experiencias de colaboración institucional y cohesión en otras regiones y ciudades e

1 Este texto resulta del proyecto PTDC/CS-GEO/105452/2008: "SPOTIA Sustainable Spatial Policy Orientations and Territorial Impact Assessment. Contribution to Portuguese context" (Orientaciones políticas de ordenación territorial sostenible y evaluación del impacto territorial. Contribución para el contexto portugués). Grupo de investigación: Modelación, Ordenamiento y Planeamiento Territorial (MOPT), del Centro de Estudios Geográficos de la Universidad de Lisboa (CEG-UL), Instituto de Geografía y Ordenamiento del Territorio (IGOT), Universidad de Lisboa, Portugal. Ente financiador del proyecto: Fundación para la Ciencia y la Tecnología FCT.

2 PhD. en Geografía Humana, Universidad de Lisboa. Profesora auxiliar, Instituto de Geografía y Ordenación Territorial (IGOTUL) ey investigadora, Centro de Estudios Geográficos de Universidad de Lisboa. Portugal. margaridav@campus.ul.pt 
identificar cuáles son los desafíos de los instrumentos de política territorial con incidencia en las áreas urbanas para el futuro.

Palabras clave: desarrollo urbano sostenible, cohesión territorial, Política de Ciudades Polis XXI - asociaciones para la regeneración urbana, Portugal.

\section{Abstract}

This paper reflects on sustainable urban development policies in Europe and their reflections in Portugal. Focusing on Portuguese Polis XXI Policy, an instrument that follows recent orientations of territorial and urban policy in Europe, it seeks to demonstrate that this is an innovative and multidimensional instrument of sustainable urban development, also capable of stimulating interactive processes of coordination and aggregation of actors around common objectives and projects (governance arrangements), noting that spatial planning policies are tending towards institutionalization, cooperation and territorial cohesion. Based on the theoretical reflections about the evolution of European spatial policy and urban policy documents, the methodology applied in this research seeks to interpret its rationale, objectives and guidelines. These inform the theoretical reflections on returning power to the cities and regions. Thus, from a selection of experiences of the implementation of the Polis XXI Policy in Portugal, we seek to find out what are the effects and which aspects are crucial for knowledge transfers, for institutional collaboration experiences, and reinforcing cohesion in other regions and cities and also identify future challenges for regional policy instruments which impact urban areas.

Keywords: sustainable urban development, territorial cohesion, Policy for Cities Polis XXI - partnerships for urban regeneration, Portugal. 


\section{Introducción}

En Europa, los aspectos del enfoque integrado o transversal relacionados con el desarrollo sostenible se basan en la "Estrategia de Gotemburgo" (COM, 2001). La referida estrategia propone medidas para enfrentarse a amenazas como el cambio climático, la pobreza, la exclusión social y el envejecimiento de la población, así como otra serie de orientaciones en materia de salud pública y transporte. Iniciada en 2004, la revisión de la mencionada estrategia, se adoptó en la Estrategia de la UE para un desarrollo sostenible (EDS) en 2006. En esta, las entidades europeas se dan cuenta de que el crecimiento económico, la cohesión social y la protección del medio ambiente deben ir de la mano, y para eso se necesita de un liderazgo político y una amplia participación pública.

Siguiendo las mismas preocupaciones, el Sexto Programa de Acción Comunitario en Materia de Medio Ambiente de la Unión Europea instaba a que se elaborara una "estrategia temática para el medio ambiente urbano", la cual se formula en 2005 (COM, 2005). En la Estrategia Temática para el Medio Ambiente Urbano se afirmaba que los retos medioambientales a los que se enfrentan las ciudades tienen importantes repercusiones en la salud y en la calidad de vida de sus habitantes, pero también en el rendimiento económico de estas mismas ciudades. Este documento de política reconoce que las actuaciones locales más exitosas son las que se valen de enfoques integra- dos para la gestión del entorno urbano. Además, la aplicación del principio de subsidiariedad, según el cual la actuación debe realizarse en el nivel más eficaz, también supone que esta se inscriba en el plano local (COM, 2005). Estas medidas, apuntadas desde la UE, tienen por objeto contribuir a una mejor aplicación de la actual política territorial y legislación medioambiental de la UE a nivel local, prestando apoyo a las autoridades locales para adoptar un enfoque más integrado de la gestión urbana. La estrategia temática (COM, 2005) incita también a una estrecha coordinación entre diversas políticas e iniciativas y una mayor colaboración entre distintos niveles de la administración.

Con más del $70 \%$ de europeos viviendo en zonas urbanas, las ciudades están en el centro de las intervenciones de la política de cohesión que tratan de explotar las capacidades económicas de Europa. Como el desarrollo económico solo puede ser sostenible si va acompañado de medidas destinadas a reducir la pobreza, la exclusión social y los problemas medioambientales, el enfoque integrado de la gestión urbana no sólo desea fomentar el crecimiento y el empleo en toda Europa, sino que también persigue objetivos sociales y medioambientales (Queirós, 2010³).

Actualmente, en toda Europa hay un interés creciente por la exploración de

3 Experiencias exitosas de políticas públicas en las áreas urbanas que buscaban integrar el desarrollo económico, la inclusión social y la calificación ambiental, se encuentran en Montreal, Canadá, con las RUI. 
formas eficaces para aplicar políticas urbanas sostenibles, promovidas en grande parte por la Dirección General de Política Regional de Unión Europea (DG Regio). Además, las sucesivas presidencias de la UE reconocen la importancia de las cuestiones urbanas y han diseñado una nueva cultura de cooperación en materia de asuntos urbanos con la Comisión Europea, el Parlamento Europeo, el Comité de las Regiones y otras instituciones europeas. A tal efecto, los ministros se han comprometido a apoyar el desarrollo de unas ciudades y regiones europeas fuertes, basándose en dos tipos de estrategias (Carta de Leipzig sobre Ciudades Europeas Sostenibles, 2007): recurrir más a los enfoques a favor de una política integrada y colaborativa de desarrollo urbano.

Aunque no existe una base jurídica para las políticas urbanas y el ordenamiento territorial en los Tratados constitutivos de la Unión Europea, esta ha elaborado un buen número de documentos políticos, ha puesto en marcha programas y iniciativas para apoyar la sostenibilidad de las ciudades, la regeneración urbana, la innovación en la política urbana y el intercambio de experiencias y buenas prácticas, como la iniciativa comunitaria URBAN (DG Regio, 2009).

Haughton, Allmendinger, Counsell y Vigar (2010) anotan que en los desarrollos de política urbana expresados en los documentos y orientaciones oficiales de UE, se observa un fomento de la experimentación de políticas, la introducción de elementos de cooperación y una contribución a un enfoque más integrado del desarrollo. Para Rydhin y Falleh (2006), en muchos y diferentes niveles y escalas, las organizaciones se han combinado en redes -más o menos complejas- con la finalidad de abordar el problema de cómo promover la acción colectiva para la gestión del desarrollo urbano y presentar un análisis detallado del funcionamiento de sus mecanismos institucionales.

Con un objetivo similar, este artículo reflexiona, a partir de documentos más relevantes de política europea para el desarrollo urbano sostenible, sobre su racionalidad, objetivos y orientaciones, y se concluye en el sentido de Haughton, Allmendinger, Counsell y Vigar (2010). Y para un análisis más concreto, se utiliza una selección de experiencias resultantes de la aplicación del instrumento Política de Ciudades Polis $X X I$, en Portugal (que sigue las orientaciones de la política europea), que evidencian iniciativas integradas de colaboración institucional, de conectividad y de cohesión territorial para un desarrollo urbano sostenible. Con esto se identifican los desafíos para el futuro para los instrumentos de política territorial con incidencia en las áreas urbanas. Se concluye, como advierten Salet, Thornley, Kreukels y Anton (2005), y Faludi (2010), que el futuro se construye ligando las diferentes entidades urbanas e intereses a través de la conectividad organizada y con esta se refuerzan la capacidad institucional y la cohesión territorial. 
1. Orientaciones de política territorial europea: cohesión territorial, complementaria de la cohesión social y económica

A pesar de todos los esfuerzos desarrollados por la UE para promover el desarrollo urbano sostenible, sucesivos Informes de Cohesión (Comisión Europea, 2011) identifican graves disparidades económicas y sociales en distintas escalas geográficas, exacerbadas por las rondas de ampliación de la UE, lo que explica una creciente percepción de que algún tipo de justicia espacial, o la solidaridad basada en las geografías europeas, se debería promover. De acuerdo con Haughton, Allmendinger, Counsell y Vigar (2010), y Morphet (2011), la cohesión territorial se va poco a poco involucrando como un objetivo esencial de cohesión.

De hecho, en 1999, los ministros de la UE responsables de la ordenación territorial y el desarrollo regional, adoptaron la Perspectiva Europea de Ordenación del Territorio, en Potsdam, conocida más recientemente como Estrategia Territorial Europea (en inglés, ESDP, European Spatial Development Perspective). La Estrategia Territorial Europea (ETE) es un marco de referencia común para un territorio europeo sin fronteras interiores, una herramienta de coherencia territorial para las políticas sectoriales y de maximización de su eficacia (Aliseda \& Muñiz, 2003; Faludi, 2010). Este instrumento desarrolla considerables acciones en los años posteriores, como son los informes de la Comisión
Europea (Ballesta \& Melikhova, 2011). El último de ellos, el quinto informe: Invirtiendo en el futuro de Europa sobre la cohesión económica, social y territorial, expone los avances de la política territorial (Comisión Europea, 2011).

ETE también supuso la puesta en marcha de programas de cooperación transnacional, por ejemplo, como destaca Morphet (2011), el INTERREG (introducido en 1990), centrado en proyectos de desarrollo territorial y que fue la palanca de la creación del Observatorio en Red de la Ordenación del Territorio Europeo (ESPON), y en 2007 llevó, además, a un nuevo marco importante en la política de ordenación del territorio en Europa, la adopción de la Agenda Territorial de la UE, en Leipzig (Territorial Agenda, 2007).

Farinós (2008, p. 13) destaca ESPON 2.3.2 como un proyecto con objetivo específico, el territorio: este se torna sujeto y objeto de gestión y decisión de los asuntos públicos. En este contexto evolutivo, se han conferido nuevos conceptos y enfoques de trabajo, a tal punto que se habla de una "nueva cultura del territorio" en las políticas públicas (Plaza, Romero \& Farinós, 2003).

Con su recomendación de adoptar una política integrada de desarrollo territorial, la Agenda Territorial de la UE se propone aprovechar el potencial de las regiones y ciudades europeas para el crecimiento económico sostenible y la creación de empleo. Una de las prioridades de la Agenda es reforzar el desarrollo equilibrado y la innovación por 
medio de la creación de redes de ciudades y ciudades-regiones. El primer Programa de Acción para la Aplicación de la Agenda Territorial (First Action Programme, 2007) se adoptó en el Consejo Informal de Ministros responsables de la ordenación del territorio y el desarrollo urbano, en las Azores, en noviembre de 2007. Faludi (2009, p. 32) considera que llegar a un acuerdo sobre la Agenda Territorial ha sido un gran logro, porque estrictamente hablando, la UE no tiene territorio, porque la UE no es un Estado.

En 2009, en la Resolución del Parlamento Europeo, de 24 de marzo (sobre la dimensión urbana de la política de cohesión en el nuevo periodo de programación), se reconoce que las zonas urbanas desempeñan un papel esencial para la aplicación eficaz de las estrategias de Lisboa y Gotemburgo y se consideran prioritarias en la política de cohesión. Esta resolución señala la importancia de un enfoque integrado con respecto a la planificación urbana y propone que el apoyo público al desarrollo urbano deba basarse en planes de desarrollo urbano integrado. Considera además que las estructuras de gobernanza existentes en los Estados miembros siguen siendo poco adecuadas para fomentar la cooperación horizontal, e insta a la Comisión a que promueva el principio de una estructura de gestión intersectorial.

El concepto de "cohesión territorial" en sí, fue mencionado por primera vez a nivel europeo en el informe Regiones y Territorios en Europa. Igualmente, el Tratado de Lisboa (2007) ha añadido la cohesión territorial a los objetivos de cohesión económica y social: una competencia compartida entre la Comisión y los Estados miembros y un especial foco en el papel de las ciudades (Mirwaldt, McMaster \& Bachtler, 2008).

Con el fin de realizar un seguimiento de la política de cohesión, se publicó el $L i$ bro Verde sobre la cohesión territorial, convertir la diversidad territorial en un punto fuerte (COM, 2008). Y en 2008 se puso en marcha un amplio debate sobre la "cohesión territorial", con el fin de ampliar los conocimientos sobre el concepto en sí mismo y sus implicaciones para la política, la cooperación y la coordinación (DG Regio, 2009). Morphet (2011) entiende que la cohesión territorial funciona a través del poder de la diferencia y acepta que para lograr fines beneficiosos para cualquier área, se necesitan diferentes enfoques, lo cual sugiere una relación contractual entre lugares y escalas y en el uso de los recursos específicos dentro de una estrategia global y un marco de colaboración.

A la par de las preocupaciones con el desarrollo sostenible, la cohesión se ha convertido por lo tanto en uno de los referentes fundamentales del proceso de integración comunitaria (Plaza, Romero \& Farinós, 2003). Pero, la 'cohesión territorial' es un concepto ampliamente criticado porque tiene, al menos, cuatro interpretaciones diferentes: i) un desarrollo policéntrico y endógeno, con el objetivo de cultivar varios clusters de la competitividad y la innovación en toda Europa; ii) un modelo de desarrollo equilibrado, con el objetivo principal 
de reducir las disparidades socioeconómicas y evitar desequilibrios; iii) la cohesión del territorio a veces se formula en términos de accesibilidad, es decir, la ambición para que los ciudadanos tengan igual acceso a las instalaciones, servicios y conocimientos, independientemente de donde vivan; iv) una forma de trabajo en red, que haga énfasis en las conexiones físicas e interactivas que existen entre los diferentes centros de comunicación y que también los vinculen con sus alrededores (Mirwaldt, McMaster \& Bachtler, 2008). Esto significa que, pese a los recientes esfuerzos de la Comisión Europea, el concepto de cohesión territorial adquiere forma pero su significado aún no ha cristalizado (Waterhout, 2008).

Sin una definición común, la 'cohesión territorial' es difícil de alcanzar, se puede abordar el concepto simplemente por las acciones de sus proponentes. Es por eso que el Libro Verde trata la necesidad de reducir las disparidades entre las diferentes categorías de ciudades y entre las ciudades ubicadas en regiones con diferentes tasas de desarrollo socioeconómico (COM, 2008; DG Regio, 2009). Este documento propone estrategias para la concentración, la conexión de los territorios y la cooperación territorial, a diversos niveles (horizontal y vertical), y también admite importantes cuestiones para un debate (la Comisión invita a todas las partes interesadas a hacer observaciones).

Se puede comprender que el Libro Verde procura un enfoque para reducir las desigualdades territoriales en la UE. De hecho, Vale (2009) señala que en la UE, las desigualdades regionales son bien conocidas y han ido en aumento en los últimos años, estableciendo un núcleo formado por el llamado "pentágono", o sea, un área poligonal cuyos vértices son Londres, París, Milán, Múnich y Hamburgo, y el resto del espacio europeo. El núcleo europeo contiene las actividades de los conocimientos más avanzados intensivos y de mayores recursos humanos calificados $\mathrm{y}$ ha sido favorecido por importantes inversiones en infraestructuras evolucionadas que tienden a perpetuar el crecimiento y dar lugar a mayores niveles de competitividad. Este espacio europeo desigual ha sido una preocupación política, especialmente desde la integración de los dos países ibéricos, en 1986, y las sucesivas ampliaciones que han seguido.

Con el objetivo de reducir los desequilibrios regionales generados por el crecimiento económico del "pentágono" europeo, se considera la cohesión territorial como otro pilar importante y complementario a la cohesión económica y social (Vale, 2009). Por eso el enfoque territorial en las políticas de la UE es, sin duda, bien recibido por las regiones de la UE. En el mismo sentido se han manifestado Plaza, Romero y Farinós (2003, p. 235) cuando afirman que la política regional europea va planteando una perspectiva integrada y multisectorial de desarrollo con una expresión espacial, ya que hace referencia a menudo a territorios, problemas y dinamismos regionales específicos como las regiones industriales 
en declive, regiones de frontera, áreas de montaña, entre otras.

Muy recientemente se comentaba en Ciudades del Mañana (UE, 2011), que las ciudades son un factor clave para el desarrollo territorial de Europa. Todavía hay muchos desafíos urbanos, entre ellos, se proponen sistemas de gobernanza territorial adaptados a unas circunstancias cambiantes, teniendo en cuenta varias escalas territoriales (supra e infraurbanas), así como enfoques transectoriales y una coordinación horizontal y vertical, ya que las ciudades deben trabajar con otros niveles de gobernanza, reforzar su cooperación y su trabajo en red, internamente y con otras ciudades, para compartir las inversiones y los servicios necesarios a una escala territorial mayor (Farinós, 2008; UE, 2011).

Estas preocupaciones también están presentes en los documentos de la Agencia Europea de Medio Ambiente (EEA, 2009): la integración europea descubre que la autoridad se ha convertido en algo cada vez más disperso a través de una variedad de diferentes niveles, actores y agencias, creando una base para la gobernanza multinivel. Como consecuencia, las ciudades tratan de reforzar la acción en el ámbito local por la participación en redes de ciudades regionales más amplias y directas en el orden europeo. Sin embargo, persiste el reto de superar las acciones aisladas a nivel local y la competencia entre ciudades y entre las regiones. A pesar de los retos, la realidad es que la gestión integrada de toda
Europa sigue siendo un asunto de unos pocos pioneros. Política aislada e intereses individuales siguen amenazando el desarrollo sostenible y la calidad a largo plazo (EEA, 2009; Van Cutsem, 2010). Así, múltiples desafíos pueden observarse durante una europeización cada vez mayor de la ordenación del territorio y el desarrollo de las ciudades (Waterhout, 2008).

\section{La tercera dimensión de la cohe- sión y la "agenda urbana"}

La Comisión Europea (COM, 2010) añade a la cohesión económica y social, la "tercera dimensión", la cohesión territorial. Y especialmente significativo es que apunta a un especial énfasis del papel de las ciudades en esta dimensión. Defiende, por lo tanto, el desarrollo de una ambiciosa política urbana, donde los recursos financieros para hacer frente a los problemas urbanos se identifican con mayor claridad, $\mathrm{y}$ donde las autoridades urbanas juegan un papel más importante en el diseño e implementación de estrategias de desarrollo urbano.

Con una misma orientación, un dictamen del Comité de las Regiones (2011/C 166/07) aprueba el principio de mayor flexibilidad en la organización de los programas operativos para intervenir en el territorio a diferentes escalas. En este dictamen se pide también que se tenga más en cuenta la dimensión urbana en la política de cohesión. Este enfoque en un desarrollo urbano integrado -considerando las 
ciudades como polos de crecimiento y desarrollo para el conjunto de su región- tendrá que encontrar apoyos en los trabajos realizados en el marco de la Carta de Leipzig, dirigida a examinar el nuevo referente común de ciudad europea sostenible.

Desde la adopción del Tratado de Lisboa, en que la cohesión territorial se ha convertido en un objetivo de la Unión con la misma importancia de la cohesión económica y social, los ministros responsables de ordenación y desarrollo territorial, en cooperación con la Comisión Europea y con el aval del Comité de las Regiones, han revisado la primera Agenda Territorial de 2007 y acordado la nueva Agenda Territorial de la Unión Europea 2020 (AT 2020). En este contexto, la nueva Agenda Territorial, que refleja las conclusiones del quinto informe sobre la cohesión, define seis prioridades territoriales (Comisión Europea, 2010): promover un desarrollo territorial equilibrado y policéntrico, fomentar el desarrollo integral de las ciudades en las zonas rurales y en áreas específicas, integrar las regiones funcionales transfronterizas, afirmar la competitividad global de las regiones con base en una economía local fuerte, mejorar la conectividad territorial de las comunidades y empresas, gestionar y vincular los valores ecológicos y el paisaje cultural de las regiones.

En conformidad con el horizonte temporal de los documentos políticos más importantes hasta el año 2020, el objetivo de la AT 2020 es proporcionar orientaciones estratégicas para el desarrollo territorial, fomentar la integración de la dimensión territorial en las diferentes políticas en todos los niveles de gobierno y garantizar la aplicación de la Estrategia Europa 2020, de acuerdo con los principios de cohesión territorial (COM, 2010). AT 2020 establece una "territorialización" de la Estrategia Europa 2020, o sea, que el crecimiento inteligente, sostenible e integrador se haga considerando los territorios donde ocurre ${ }^{4}$. Teniendo en cuenta que una parte considerable de la población europea vive en ciudades, ¿cómo llevar a la práctica las prioridades de la Agenda Territorial en los territorios urbanos?

AT 2020 anima las regiones y ciudades para desarrollar redes y adoptar estrategias integradas y planes espaciales como apropiados para aumentar la eficiencia de las intervenciones en el territorio. Se enfatiza que cuando las ciudades más desarrolladas y las regiones de Europa cooperan como partes de un modelo, añaden valor y actúan como centros que contribuyen al desarrollo de sus regiones más amplias. Además, la política de desarrollo territorial debe fomentar la competitividad territorial del espacio de la UE también fuera de la zona del pentágono.

4 Salir de una profunda crisis, reducir el desempleo y la pobreza, al tiempo que impulsa una economía baja en carbono, es una tarea para Europa, motivo por el cual el Consejo Europeo adoptó la estrategia Europa 2020 (COM, 2010, 2020 final). Europa 2020 es una visión de la economía social de mercado para este continente, que propone objetivos y tres prioridades, en 2020, que se refuerzan mutuamente: crecimiento inteligente, crecimiento sostenible y crecimiento integrador. 
Se recomienda además aplicar un enfoque integrado y de varios niveles en el desarrollo urbano y políticas de regeneración. La cooperación y trabajo en red de las ciudades puede contribuir a un desarrollo inteligente de las regiones de la ciudad a diferentes escalas a largo plazo.

La Agenda recomienda que las iniciativas de cooperación territorial deban orientarse a los objetivos de la construcción de la cohesión territorial en la experiencia de la antigua iniciativa comunitaria INTERREG y los programas actuales transnacionales, y pone de relieve el apoyo de los instrumentos europeos por el intercambio de buenas prácticas, así como proyectos de innovación y transferencia de conocimientos entre las organizaciones participantes, como la creación de redes y programas interregionales (INTERREG, INTERACT, URBACT, etc.).

INTERREG, con recursos financieros limitados, ha apoyado más de dos centenas de programas transfronterizos y se ha expandido y desarrollado en más de cuatro períodos de financiación (1990-93, 1994-99, 2000-06, 200713), y ha convertido, además, la cooperación territorial -cooperación transfronteriza, transnacional e interregional- en uno de los tres objetivos de los Fondos Estructurales (Mirwaldt, McMaster \& Bachtler, 2008). La cooperación territorial se ha implementado a través de este programa que ofrece nuevas soluciones para problemas de desarrollo y valiosas lecciones de cohesión territorial, que han llevado, por consiguiente, a una aceptación "casi universal" de que la cooperación es favorable a la cohesión territorial. Una de las lecciones de INTERREG es que si una definición común parece difícil de lograr, una manera de acercarse al concepto de cohesión territorial consiste en considerar los cursos de acción por la cual sus proponentes quieren conseguirla. Es por eso que la cohesión territorial refuerza la importancia del acceso a los servicios de interés general, el desarrollo sostenible, las "geografías funcionales" y el análisis territorial, (mientras que la cohesión económica y social se centra en las disparidades regionales en términos de competitividad y bienestar). Waterhout (2008) añade a estas, la idea que los proyectos INTERREG aportan un valor añadido a los procesos políticos, ya que tratan a menudo con cuestiones individuales en lugar de los sistemas complejos de actores y objetivos multidimensionales.

\section{Iniciativas integradas para el desarrollo urbano sostenible en Portugal: contribuciones para la Agenda Territorial}

El tema de la cohesión territorial ha generado un debate público en el ámbito europeo y asignado un valor añadido para su adopción en las agendas de sus Estados miembros. Todavía, afirman Santinha y Marques (2012), la adopción del principio de la cohesión territorial como un paradigma de desarrollo de la Unión Europea, no oculta una cierta ambigüedad con res- 
pecto a su concepto y funcionamiento, a pesar de su fuerte institucionalización. Las referidas autoras argumentan que en los documentos recientes de políticas públicas territoriales y ambientales en Portugal, la cohesión territorial surge, en la mayoría de los casos, asociada a la búsqueda de un desarrollo regional y urbano integrado, a la creación de redes, pero también en cuanto accesibilidad a los servicios de interés general y a la integración y articulación sectorial.

Un problema importante de la competitividad territorial en Portugal está vinculado a las debilidades del sistema urbano, que se traducen en las estrechas dimensiones demográficas y funcionales de las ciudades, en la fragilidad de su proyección, en la dispersión marcada de la infraestructura y el equipamiento, en un déficit de especialización y complementariedad interurbana y en la ausencia de una cultura de colaboración eficaz (DGOTDU, 2011). Estos son fuertes motivos para reflexionar sobre las perspectivas de la competitividad urbana, la cohesión social y la sostenibilidad del medio ambiente urbano en Portugal, retos encuadrados en las orientaciones políticas europeas. Por eso, la Política de Ciudades Polis XXI, que da un enfoque espacial destacado a las ciudades, es un programa político que utiliza el conceptual referencial de cohesión territorial y de sostenibilidad pertinente en el contexto actual de política de cohesión territorial de la UE.

Remontando un poco en el tiempo en la historia de las políticas urbanas, con el fin de responder a los desafíos cada vez más numerosos y complejos que enfrentan las zonas urbanas, Portugal ha puesto en marcha una agenda urbana para apoyar el desarrollo urbano sostenible en las últimas dos décadas. Desde los 90, PROSIURB, URBAN I y II, Intervención Operacional de Rehabilitación Urbana son buenos ejemplos ${ }^{5}$. La experiencia de estos primeros instrumentos estructurados de política urbana ha permitido identificar varios factores de éxito en el proceso de desarrollo urbano sostenible (DG Regio, 2011). Las lecciones fueron transportadas a los programas convencionales, incluyendo una intervención conocida como Programa POLIS - Programa para la Mejora de Rehabilitación y Medio Ambiente de las Zonas Urbanas, o la Iniciativa de Barrios Críticos en las Áreas Metropolitanas de Lisboa y Porto, entre otros. Con la Iniciativa de Barrios Críticos se ganó experiencia en la promoción de un enfoque territorial integrado, a través de intervenciones experimentales en torno a modelos organizativos

5 Entre los programas de intervención en las zonas urbanas, merece una especial referencia el programa PROSIURB, establecido en 1994 para apoyar el planeamiento estratégico en ciudades de tamaño medio. De acuerdo con el Gabinete Coordinador del PROSIURB (MAOT, 2000), la intervención fue dirigida a mejorar la accesibilidad y otras infraestructuras y redes de equipos, así como a la promoción de políticas que contradicen las disparidades territoriales y que evidencian la marginación y la exclusión social. La naturaleza integradora e innovadora de PROSIURB ha promovido sinergias entre las distintas instituciones y agentes, llamando la atención por la importancia de las relaciones de complementariedad entre las ciudades. PROSIURB dio, por consiguiente, un paso adelante en la innovación institucional, hacia una mayor eficiencia y flexibilidad en la construcción de la ciudad, porque ha tratado de contribuir al establecimiento de los principios fundamentales del desarrollo urbano a través de procesos de negociación. 
alternativos de regeneración urbana: coordinación estratégica, cooperación interministerial y desarrollo de planes de intervención centrados en el diagnóstico de los territorios y diseñados por el conjunto de los interlocutores locales (Sousa, 2008).

Más recientemente, presentado en 2008, la Política de las Ciudades PO$L I S X X I$ es el documento de referencia actual para la política urbana en Portugal y se inscribe para el período de programación 2007-20136. Esta es una política multi-instrumental (comprende varias herramientas) y tiene como objetivos apoyar la competitividad de la regeneración urbana, la diferenciación y la integración regional. Se estableció en torno a cuatro prioridades/instrumentos principales, que corresponden a diferentes escalas geográficas: i) asociaciones para la regeneración urbana (escala intraurbana); ii) redes urbanas para la competitividad y la innovación (escala interurbana); iii) asociaciones ruralesurbanas estructuradoras del sistema urbano nacional (escala regional); iv) acciones innovadoras para el desarrollo urbano (sin escala específica).

Una de las apuestas de esta política específica es desarrollar el sistema urbano a través de la creación de redes entre las ciudades, un concepto operativo en las redes de instrumentos de política urbana para la competitividad y la innovación, sobre la base de un modelo de gobier-

6 Más de mil millones de euros de coparticipación de UE, introducida por el Ministerio de Medio Ambiente, Ordenación del Territorio y Desarrollo Regional (DG Regio, 2011). no compartido y la complementariedad estratégica entre los diferentes factores y recursos de cada ciudad (DGOTDU, 2011). La consecución de estos objetivos requiere formas de acción y de organización que aún no están arraigados en la cultura política e institucional portuguesa.

Otro concepto incluso en este programa es la regeneración urbanat, considerado como un proceso estratégico integrado, que involucra las iniciativas pública, privada y otros actores de la ciudadanía en torno a instituciones creadas específicamente para este propósito, y que procura mejorar el capital humano, social, material, cultural y económico de los territorios urbanos necesitados.

En el ámbito de la configuración de la Política de Ciudades Polis XXI, las asociaciones para la regeneración urbana (ARU8), son un instrumento de política de regeneración urbana que se aplica a territorios urbanos específicos, para estimular la gobernanza territorial y la promoción de enfoques integrados de desarrollo urbano, así como para cubrir una amplia gama de medidas para la

7 En junio de 2010, se aprobó la Declaración de Toledo, una iniciativa de los ministros de la UE responsables del desarrollo urbano. La Declaración de Toledo se refiere a la "regeneración integral urbana", basada en los principios del desarrollo sostenible, la cohesión y la integración, ya que es la única manera de lograr una mayor competitividad económica, la eco-eficiencia, la cohesión social y el progreso en las ciudades de Europa, y de garantizar la calidad de vida de los ciudadanos presentes y futuros de Europa. La Declaración se basa en la aplicación de la Estrategia Europa 2020, la implementación de la Declaración Europea de Ciudades Sostenibles y la necesidad de consolidar la Agenda Urbana Europea.

8 En portugués: Parcerias para a Regeneração Urbana (PRU). 
mejora de las infraestructuras, la renovación del espacio público, la creación de nuevos equipamientos públicos, la rehabilitación, el desarrollo social y la mejora del medioambiente. Asociación, integración, coherencia, sostenibilidad e inclusión, son algunas palabras clave en este instrumento de política (Reis \& Pinho, 2008).

A pesar de las experiencias anteriores de intervenciones en áreas urbanas, la regeneración urbana en Portugal no ha contado con el apoyo de estos tipos de instrumentos, ni los actores han ganado profunda tradición de trabajar juntos para este propósito, por lo que, ideológicamente, las asociaciones para la regeneración urbana de la Política de Ciudades Polis XXI, se presentan como un modelo innovador de gestión urbana. Después de algunos años de aplicación, es importante evaluar el alcance de esta política, especialmente desde la intervención del $\mathrm{FMI} / \mathrm{UE} / \mathrm{BCE}$ 9 que reduce el gasto público $\mathrm{y}$, en particular el que tiene fines sociales. Así, la crisis financiera y la posterior aplicación de medidas de austeridad, condicionan todos los problemas de desarrollo urbano

9 En abril de 2011, el Gobierno portugués solicitó el apoyo financiero a la Unión Europea. A principios de mayo, el primer ministro anunció que había acordado un rescate con la Unión Europea (UE)/Banco Central Europeo (BCE)/ Fondo Monetario Internacional (FMI), por importe de 78 millones de euros. A cambio de este préstamo, Portugal accedió a una serie de medidas para aumentar los ingresos fiscales y reducir el gasto, incluida la reducción de los salarios del sector público y la privatización de las acciones de las empresas de energía nacionales (GALP, EDP y REN), transporte (Aeropuertos Portugal, TAP y la carga de la rama de los trenes de Portugal, CP), comunicaciones (Oficina de Correos de Portugal), y los seguros (Caixa Seguros), así como otras empresas más pequeñas. y las estrategias propuestas, hoy y en el futuro post-2013. Así, con base en una publicación (Ecorys \& CEDRU para la DG Regio), y otro estudio de Centro de Estudios Sociales de la Universidad de Coimbra, se seleccionaron áreas urbanas en Portugal como estudios de caso, elegidos para representar los diferentes tipos de regiones urbanas, estudiando tanto las experiencias de asociaciones para la regeneración urbana, como las formas de ejecución del desarrollo urbano (DG Regio, 2011).

En un país que está condicionado por penosas medidas externas de austeridad, la Política de Ciudades Polis XXI es un instrumento que ha iniciado un proceso de aprendizaje. Algunos aspectos cruciales basados en las ARU para transferencias potenciales de experiencias y conocimiento, se desvelan en la sección siguiente; se muestra que ellas van al encuentro de los objetivos de UE para la cohesión territorial.

\section{Política de Ciudades Polis XXI y las asociaciones para la regene- ración urbana}

En sintonía con las prioridades de la ET2020 y con la Agenda Territorial, la Política de Ciudades Polis XXI tiene como ambición tornar las áreas urbanas donde se concentra la mayoría de la población portuguesa, más competitivas, innovadoras e inclusivas, con mejor ambiente, bien planificadas y gobernadas. En la configuración general de la Política de Ciudades Polis XXI se establecieran tres prioridades/dominios/ins- 
trumentos de política, para tener financiación, que corresponden a tres escalas geográficas de intervención: "redes urbanas", "asociaciones para la regeneración urbana" y "acciones innovadoras para el desarrollo urbano/rural".

En este diseño se revela el marco reciente de la UE, la cohesión territorial, ya que trabajando en red, las ciudades y las entidades urbanas, desempeñan un papel clave en el desarrollo sostenible. $\mathrm{E}$ independientemente de las diferentes definiciones que el concepto de cohesión territorial asume, vale destacar que en los tres instrumentos se identifican orientaciones, cursos de acción y financiación para el desarrollo policéntrico con un enfoque más funcional y flexible, dando énfasis a las conexiones físicas e interactivas, al mismo tiempo que se estimulan asociaciones para disminuir los desequilibrios socioeconómicos que atentan contra la dimensión de la calidad ambiental, se incentiva el acceso a conocimientos y servicios de interés general. Es por la contribución al fortalecimiento de relaciones de cooperación entre actores y territorios para la cohesión, que la prioridad que se sintetiza en esta sección es aquella de la escala intraurbana, materializada en la dimensión de las asociaciones para la regeneración urbana.

Las asociaciones para la regeneración urbana (ARU) se conceptúan para apoyar actividades de revitalización integrada de los espacios intraurbanos, apoyados por una amplia estructura de asociación local (municipio, servicios descentralizados del gobierno central,
ONG, empresas, etc.). Este instrumento se diferencia por la elección de los espacios intraurbanos como lugares de acción. Además requiere una definición sustancial y coherente de la intervención y presupone que es crucial reunir a diferentes actores del territorio local, para tomar medidas que promuevan la integración de los aspectos materiales e inmateriales, y concebir una perspectiva a largo plazo con resultados/operaciones de valor añadido (económico, social y cultural y de la calidad del medio ambiente) (Reis \& Pinho, 2008; DG Regio, 2011).

Las ARU se articulan por programas de acción; cada programa se basa en una asociación para la regeneración urbana, entendida como una cooperación estructurada y formal entre las entidades que se propongan desarrollar e implementar el Programa de Acción Conjunta para la regeneración de una zona específica de una determinada ciudad. Una "asociación para la regeneración urbana" consiste en el municipio, que debe tomar la iniciativa para estructurar la sociedad y dirigir la preparación del Programa de Acción, y otros actores urbanos (propietarios, empresas, fundaciones, ONG, etc.).

El informe de seguimiento y evaluación de las candidaturas de los Programas de Acción, realizado por Reis y Pinho, a los instrumento ARU, trata de comprender y responder a: ¿cómo se está formulando, desde el punto de vista local, un problema territorial de regeneración urbana; ¿qué recursos institucionales y materiales se identifican para iniciar 
una asociación (dando una justificación de la acción pública inusual en el ámbito municipal y que culminó en la creación de nuevos activos en el territorio); ¿cuál es la fuerza que provoca la formación de una asociación (y la forma en que se constituye como un elemento central del programa de acción); ¿qué relación se establece entre los proyectos de intervención que componen los programas de acción (discutiendo su integración, las sinergias que crean, los objetivos de orden económico y social, la originalidad y la sostenibilidad); ¿se puede ver en ARU un factor decisivo para la creación de nuevos entornos urbanos (es decir, los nuevos usos de la ciudad que pasan también a ser vistos como un medio de uso más eficiente y hábil de los recursos ambientales, energía y transporte, de espacio público urbano, dando lugar a nuevas economías -materiales o sociales- de estos territorios).

En dicho informe se relata que aproximadamente la mitad de los casos son aquellos en que se superpone una "asociación de regeneración”, lo que en otras circunstancias serían "proyectos independientes", sin cambio de proporciones entre los actores o entre las dimensiones físicas e inmateriales o la creación de procesos que hacen la visión de conjunto de la ciudad y la intervención integral. En algunos casos, la identificación del problema está particularmente bien hecha, o sea, la intervención propuesta deriva directamente del problema y también se asocia directamente con los caminos de su superación. Hay casos en que las asociaciones han servido para generar una nueva actividad en un centro urbano: en ellas se reúnen los distintos socios y con la regeneración urbana se provoca una base directa para la generación de nuevas soluciones para optimizar el área de intervención. Pero, menos positivo, es que en la mitad de los casos se conjuga el trabajo de los socios singulares realizados bajo el mismo programa de acción.

Las asociaciones son el dispositivo principal (una condición definitoria normativamente) de este instrumento de política. En el pasado hubo poca acumulación de experiencias en asociaciones estratégicas colectivas para actuar en las zonas urbanas, con la excepción de PROSIURB en las ciudades medias (y de la intervención excepcional conocida como Expo'98 en Lisboa). Hay cuestiones de formación, incluida la falta de pericia en algunas ciudades, y la falta de oportunidades para la creación de redes con el fin de transferir el conocimiento y promover el aprendizaje (DG Regio, 2011). Además no se ha madurado suficientemente un capital social disponible para su uso con el instrumento ARU. Por eso Reis y Pinho (2008) arguyen que la idea de asociación, el elemento central de generación de "tensión creativa", tuvo en el actor público (es decir, las autoridades locales) su plataforma más importante.

Los diferentes tipos de actores que participan en las ARU, reconocidos por Reis y Pinho (2008), son las municipalidades, empresas municipales, promotores inmobiliarios, universidades, asociaciones de economía social, animación o la promoción de contextos culturales para 
los negocios. Se enfatiza así la convergencia institucional: la superación de la situación de una sola entidad, la autoridad local, para una cooperación entre socios institucionalizada por una ARU.

El informe revela que en aproximadamente la mitad de los casos que han pretendido utilizar el instrumento ARU, a los recursos que han permitido la intervención urbanística municipal se adicionaron capacidades de otros actores, lo que facilitó la intervención, influyendo en el programa de acción. Pero se han revelado situaciones en que los recursos movilizados por la asociación establecen medios innovadores de intervención y nuevos activos urbanos. Ocurre también que las intervenciones físicas constituyen una base fundamentada, aunque muchas veces dispersa, para los objetivos materiales económicos o sociales, pero estos se establecen de manera excesivamente amplia (Reis \& Pinho, 2008). El recurso que se movilizó más, fue la capacidad de intervenir físicamente en los edificios municipales y espacios públicos.

En términos generales, hay propuestas que se iniciaron a partir de la identificación previa de un problema de exclusión social y decadencia urbana, y hay otras que privilegian las cuestiones de competitividad, en el supuesto de que era importante para impulsar la economía.

Los problemas de accesibilidad y movilidad y las cuestiones energéticas son relevantes en las causas de los proyectos. Reis y Pinho (2008) afirman que cerca del $40 \%$ de las solicitudes demuestra una preocupación clara y consistente con el tema de la sostenibilidad del medio ambiente, tanto en su dimensión energética y biofísica, como en la forma en que favorecen el cambio del "paradigma de la movilidad" por el "paradigma de la accesibilidad", lo que significa que la asociación viabiliza la integración de los medios más suaves de los viajes en la vida urbana cotidiana. Todavía en $60 \%$ de los casos, si bien hay preocupaciones ambientales, las propuestas o son triviales, con impacto reducido en la calidad del ambiente urbano, o las preocupaciones no se reflejan en las propuestas presentadas.

El instrumento ARU demuestra que la acción pública para mitigar los problemas asociados con el desarrollo urbano sostenible debe incluir la adopción de modelos más descentralizados, dado que la eficacia de las operaciones relacionadas con la descentralización hace posible combinar las acciones locales que son más capaces de movilizar el conocimiento local como un recurso y más eficaces con las actividades del Estado central. Esto significa que, en el caso de las zonas urbanas, especialmente afectadas por la pobreza y la exclusión social, es particularmente importante que las políticas actuales, las iniciativas y formas de integración se adapten al contexto local (Queirós, 2010).

La evaluación hecha por Ecorys y CEDRU para DG Regio (2011, p. 13) del instrumento ARU, reconoce que los tres primeros años de aplicación de este instrumento relevaron desafíos a su funcionamiento, entre ellos: retrasos, 
dificultad de acceso a instrumentos financieros, poca participación del sector privado, falta de conocimiento, bajos niveles de participación de la comunidad y del gobierno central, necesidad de una mayor supervisión y evaluación. Por estos desafíos, como en los programas de política más recientes, las organizaciones involucradas en la ejecución de acciones se han enfrentado a obstáculos que limitan la eficacia de la realización de los programas, así como su alcance (DG Regio, 2011).

\section{Lecciones de la experiencia}

El instrumento de política pública -Política de Ciudades Polis XXI- particularmente en las ARU, es simultáneamente experimental, innovador y multidimensional. Puede todavía comportar una ambición mayor que la "masa crítica" o la "capacidad técnica" instalada (DG Regio, 2011). De hecho, es mucho lo que se pide con este enfoque de "abajo-arriba" (bottom-up approach): se pone un fuerte énfasis en las asociaciones en el desencadenamiento de proyectos con lógicas de inclusión social, de sostenibilidad económica y financiera, y en su naturaleza integrada. Si, por una parte, la tradición de política local (municipal) en áreas urbanas ha estado muy basada en las actuaciones aisladas y no integradas de los actores públicos y de la sociedad civil, dirigidas a intervenciones de regeneración física (material) de los territorios, por otra parte, la lógica del gobierno para aprobación de los proyectos (de infraestructuras, de equipamientos, etc.) se pauta mucho más por la redistribución territorial, que por lógicas limitadas a criterios de eficiencia. Es por eso que, como afirman Reis y Pinho (2008), solo un proceso cumulativo de aprendizaje puede romper con el padrón de proyectos aislados posibilitando lógicas en asociación, proyectos articulados con realizaciones materiales y cada vez más con objetivos inmateriales o sociales. Ese proceso ha empezado.

¿Qué se aprende con las ARU? En primer término, se identifican algunas dificultades, como el establecer una visión del contenido de los proyectos, es decir, los nuevos efectos que desencadenan, al mismo tiempo que se señalan los peligros de constituir asociaciones no sostenibles. De hecho, las asociaciones que sustentan los proyectos son lo más difícil de lograr, dada la fragilidad de una tradición establecida con anterioridad de colaboración en estos temas. Reis y Pinho (2008) dicen que el grado de participación efectiva de los nuevos actores es muy diverso. En particular, parece que la participación de los actores cuya esfera de acción es tradicionalmente más alejada del núcleo de las políticas urbanas, es más difícil de asegurar. Se cree que en esto es donde hay posibilidad de lograr avances en el aprendizaje.

En segundo término, se percibe como muy positivo el haberse establecido una agenda regional (encuadrada por una política integrada a nivel nacional) de políticas públicas para la regeneración urbana; el haberse creado un terreno adecuado para la concertación local y la convergencia de los actores 
para solucionar problemas complexos colectivos; el hecho de profundizar la idea de sostenibilidad como un problema colectivo de gestión urbana y de demostrar la necesidad de una visión holística de la ciudad en lugar de una visión materialista y atomizada de las obras públicas; el haberse permitido la elección de las unidades territoriales con geometría variable para las intervenciones, y buscado la regeneración urbana para la inclusión social.

Al final, se percibe el potencial de las ARU como motor del empoderamiento de las instituciones locales para la gobernanza, como estímulo a la transparencia de los procesos y a la diversificación de los actores involucrados en acciones colectivas. Todo indica que el concepto alternativo de "desarrollo como libertad" (una noción de Amartya Sen) está basado en las ARU, ya que este instrumento proporciona a los actores la capacidad de amplificar y desarrollar sus competencias, la opción de elegir los temas y las intervenciones para un desarrollo sostenible, en los territorios en el que viven o actúan.

En la UE, se vive hoy una época de cambios de prioridades políticas y en ellas, la calidad de vida en los territorios urbanos, es una preocupación creciente de sus instituciones. La Política de Ciudades Polis XXI, particularmente en su instrumento ARU, se identifica, por lo menos, con uno de los objetivos de Europa 2020, o sea, da un impulso (a través de un instrumento de política territorial) al crecimiento integrador.
La incorporación de la tercera dimensión de la política regional, la cohesión territorial, coloca el énfasis en el potencial de instrumentos de política urbana -como las ARU- para cooperación territorial en las áreas urbanas, conciliación de intereses y desarrollo de redes de cooperación de actores, integración en el territorio de proyectos materiales e inmateriales, etc. Representa un proceso en el que se coordinan los aspectos espaciales, sectoriales y temporales de las áreas clave de la política urbana (Carta de Leipzig, 2007, p. 3). En este sentido, las autoridades locales con sus asociados definen las prioridades de desarrollo, mientras que las reglas generales se establecen a nivel nacional y europeo. Así, la arquitectura inteligente de instrumentos como las ARU, permite una definición común de regeneración urbana y de cohesión territorial, pero no de una talla única para toda la solución (no one-size-fits-all solution).

A fin de lograr los objetivos de cohesión e integración social en las ciudades y áreas urbanas, se resalta la importancia de experiencias y conocimientos para apoyar los actores implicados en el desarrollo urbano, con el objetivo de reforzar la dimensión urbana de la Estrategia para el Medio Ambiente Urbano, la Estrategia de Lisboa, la Carta de Leipzig, la Agenda Territorial, la Estrategia Europa 2020, y otros instrumentos de política para, a través de una conectividad organizada, apoyar ciudades que sean fuertes y en las que se viva bien. Esto lo anticipaba Farinós (2008, p. 16) cuando afirmó que cada territorio identifica su propio potencial de desarrollo 
y su posición en el territorio europeo, y enmarca sus estrategias de desarrollo territorial en un contexto de desarrollo transnacional y europeo.

\section{Literatura Citada}

Aliseda, J. M. \& Muñiz, M. P. (1996-2003). La estrategia territorial europea (ETE): una concepción integradora y cohesionada del espacio. Norba. Revista de Historia, 16, 701-707.

Ballesta, J. A. C. \& Melikhova, Y. (2011). Perspectiva territorial de la unión europea: el largo camino hacia la cohesión territorial. Cuadernos Geográficos, 47 (2010-2), 169-188.

Carta de Leipzig (2007). Recuperado de http://www.fomento.gob.es/MFOM/ LANG_CASTELLANO/DIRECCIONES_GENERALES/ARQ_VIVIENDA/ SUELO_Y_POLITICAS/ACTIVIDAD/CARTA_LEIPZIG/

COM (2001). Estrategia de Gotemburgo. Desarrollo sostenible en Europa para un mundo mejor: estrategia de la Unión Europea para un desarrollo sostenible.

COM (2005). Estrategia temática para el medio ambiente urbano. Comunicación de la Comisión al Consejo y al Parlamento Europeo, de 11 de enero de 2006.

COM (2008). Livro Verde sobre a Coesão Territorial Europeia Tirar Partido da Diversidade Territorial. Comunicação da Comissão ao Conselho, ao Parlamento Europeu, ao Comité das Regiões e ao Comité Económico e Social Europeu.

COM (2010a). Europa 2020: una estrategia para un crecimiento inteligente, sostenible e integrador. Communication from the Commission Europe 2020: A strategy for smart, sustainable and inclusive growth.

COM (2010b). Conclusions of the fifth report on economic, social and territorial cohesion: the future of cohesion policy. Communication from the Commission to the European Parliament, the Council, the European Economic and Social Committee, the Committee of the Regions and the European Investment Bank.

Comisión Europea (2010). Invirtiendo en el futuro de Europa. Quinto informe sobre la cohesión económica, social y territorial. Bruxelas: UE.

DG Regio (2009). Fomentar un desarrollo urbano sostenible en Europa. Logros $y$ oportunidades. Unión Europea. Política Regional. Comisión Europea Dirección General de Política Regional. Recuperado de http://ec.europa.eu/ regional_policy/index_es.htm. 
DG Regio (2011). Desenvolvimento urbano sustentável em Portugal: uma abordagem integrada. Relatório para a Direcção-Geral da Política Regional da Comissão Europeia. Lisboa: Ecorys UK \& CEDRU.

DGOTDU (2011). Contributo da DGOTDU para o $2^{\circ}$ Relatório bienal da ENDS. Documento Técnico DGOTDU 06/201. Lisboa: DGOTDU, MAOT.

EEA (2009). Ensuring quality of life in Europe's cities and towns. Tackling the environmental challenges driven by European and global change. EEA Report no5 (2009). Denmark: European Environment Agency.

Faludi, A. (2009). A turning point in the development of European spatial planning? The 'Territorial Agenda of the European Union' and the 'First Action Programme'. Progress in Planning, 71, 1-42.

Faludi, A. (2010). Cohesion, Coherence, Cooperation: European Spatial Planning Coming of Age? London: Routledge.

Farinós, J. (2008). Gobernanza territorial para el desarrollo sostenible: estado de la cuestión y agenda. Boletín de la A.G.E., 46, 11-32.

First Action Programme (2007). First Action Programme for the Implementation of the Territorial Agenda of the European Union. Agreed on the 23rd November 2007 at Ponta Delgada, Azores. Portuguese Presidency. Retrieved from http://www. eu-territorial-agenda.eu/Reference\%20Documents/AP1_23NovembroVfinal. pdf.

Haughton, G., Allmendinger, P., Counsell, D. \& Vigar, G. (2010). The New Spatial Planning. Territorial management with soft spaces and fuzzy boundaries. London: Routledge.

MAOT (2000). Avaliação geral do PROSIURB. Objectivos estratégicos, concertação, parcerias e domínios de intervenção. Lisboa: Gabinete Coordenador do PROSIURB. Lisboa: Ministério do Ambiente e Ordenamento do Território (MAOT).

Mirwaldt, K., McMaster, I. \& Bachtler, J. (2008). Reconsidering Cohesion Policy: The Contested Debate on Territorial Cohesion. EoRPA paper 08/5. European Policies Research Centre. United Kingdom: University of Strathclyde Glasgow.

Morphet, J. (2011). Effective Practice in Spatial Planning. London: Routledge.

Plaza, J. I., Romero, J. \& Farinós, J. (2003). Nueva cultura y gobierno del territorio en Europa. Ería, 61, 227-249. 
Queirós, M. (2010). Integrated Urban Revitalization in Montreal: lessons from local development initiatives. Finisterra, Revista Portuguesa de Geografia, XLV, (89), 47-77.

Reis, J. \& Pinho, P. (2008). Política de Cidades Polis XXI. Parcerias para a Regeneração Urbana. Relatório de Monitorização. Coimbra: CES / FEUC.

Rydin, Y. \& Falleth, E. (eds.) (2006). Networks and Institutions in Natural Resource Management. Cheltenham, UK: Edward Elgar.

Salet, W., Thornley, A. \& Kreukels, A. (eds.) (2005). Metropolitan Governance and Spatial Planning. London: Spon Press.

Santinha, G. \& Marques, T. (2012). A integração do principio de Coesão Territorial na agenda política: o caso português. Revista de Geografia e Ordenamento do Território, (2), 215-244.

Sousa, S. (2008). Iniciativa Bairros Críticos: Uma experiência em torno de modelos de governança na gestão do territorio. Cidades - Comunidades e Territórios. Centro de Estudos Territoriais, (16), 69-75. Lisboa.

Territorial Agenda (2007). Territorial Agenda of the European Union towards a More Competitive and Sustainable Europe of Diverse Regions. Informal Ministerial Meeting on Urban Development and Territorial Cohesion in Leipzig, on 24/25 May 2007. Retrieved from http://www.eu-territorialagenda.eu/Reference\%20Documents/Territorial-Agenda-of-the-EuropeanUnion-Agreed-on-25-May-2007.pdf.

UE (2011). Ciudades del Mañana. Retos, visiones y caminos a seguir. Unión Europea. Política Regional. Recuperado de http://ec.europa.eu/regional_ policy/conferences/citiesoftomorrow/index_en.cfm.

Vale, M. (2009). Regional Survey: EU Territorial Cohesion Green Paper: Member States' Perspectives. The EU's Territorial Cohesion Policy. Regions. The Voice of the Membership. Regional Studies Association, (276), 10.

Van Cutsem, M. (2010). Cities of tomorrow. Directorate General for Regional Policy. Wallonia, Belgium: Foresight Unit, The Destrée Institute.

Waterhout, B. (2008). The institutionalization of European Spatial Planning. The series Sustainable Urban Areas. TU Delft, The Neederlands: IOS Press. 
Recepción: 15 de Julio de 2012.

Evaluación: 30 de octubre de 2012.

Aprobación: 20 de febrero de 2013. 